\title{
Kinetic Features of Formation of Supramolecular Matrices on the Basis of Silica Monodisperse Spherical Particles
}

\author{
D. Kamashev ${ }^{(\bowtie)}$ \\ Institute of Geology Komi SC UB RAS, Syktyvkar, Russia \\ kamashev@geo.komisc.ru
}

\begin{abstract}
We have established that under such conditions, when the formation of a supramolecular structure from spherical silica particles $220-320 \mathrm{~nm}$ in diameter is limited by the rate of introduction of particles into the sedimentation zone (sedimentation deposition in a constant cross section tube), the particle deposition rate, as well as the formation rate of the supramolecular structure, is strictly linear. At the same time, under conditions of excess of disperse phase in the zone of formation of the supramolecular structure (sedimentation deposition in a tube with a modified cross section), the deposition rate is also linear, but there is some delay in formation of the supramolecular structure in time, the larger the smaller is the particle size of the disperse phase. After the formation of the supramolecular structure is completed, a region with an increased concentration of the disperse phase remains, the height of which is greater, the smaller is the particle size. It is shown that a certain concentration of the disperse phase is necessary to begin the formation of a supramolecular structure, below which the formation of a supramolecularly ordered structure does not occur. The concentration of the disperse phase, necessary for beginning of formation of a supramolecular structure, is a constant value that does not vary with time and depends only on the size of the particles.
\end{abstract}

Keywords: Supramolecular structure $\cdot$ Monodisperse spherical silica particles

\section{Introduction}

The supramolecularly ordered structures, based on monodisperse spherical silica particles, generated interest relatively long ago, back in the 70 s of the last century, in connection with attempts to synthesize artificial analogs of noble opal on their basis (Stober et al. 1968). The overwhelming majority of studies of that time were aimed at developing conditions for synthesis of spherical silica particles and supramolecular structures based on them, and ended with the development of methodological bases for production of synthetic noble opals (Deniskina et al. 1987). However at present the supramolecular silica particles are more often considered as promising objects for synthesis of new composition materials, photonic crystals and nanostructured materials based on them. The 3D ordered closest packing of monodisperse silica spheres is an ideal matrix for creating a wide class of new nanostructured materials, but it greatly increases requirements for the monodispersity of particles, their size and the defects of 
derived supramolecular structures (Kamashev 2012). With this aim and within the task to develop the basis of synthesis of supramolecularly ordered matrices we carried out experimental studies of the rate of precipitation of silica particles and formation of a supramolecular structure on their basis in various conditions (growth modes).

\section{Methods and Approaches}

Monodisperse spherical silica particles with radius 109, 138 and $158 \mathrm{~nm}$ were synthesized by Stober-Fink method (Stober et al. 1968). The sizes of the obtained particles were determined by Photocor Complex dynamic light scattering spectrometer at a laser wavelength $661 \mathrm{~nm}$, a scattering angle $90^{\circ}$, and a correlation function accumulation time $20 \mathrm{~min}$. The initial concentration of the dispersed phase particles was about 2 wt.\%. The deposition rate of the silica particles was measured in glass tubes $750 \mathrm{~mm}$ high and $20 \mathrm{~mm}$ in diameter, with the control of deposition boundary advancing every 30 days with accuracy $0.5 \mathrm{~mm}$. The average daily temperature was also taken into account to calculate the temperature correction associated with the expansion of the dispersion environment. The following values were obtained for the silica particles of different radius (Fig. 1).

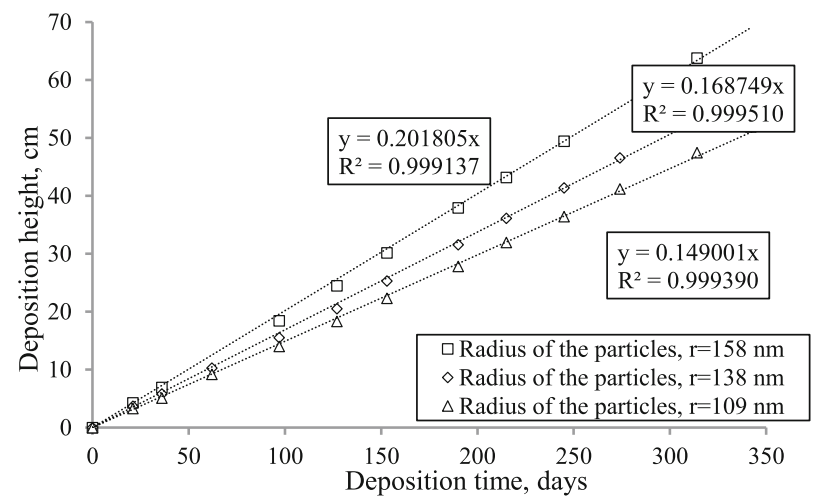

Fig. 1. Dependence of height of deposition of silica particles of different sizes over time. The coefficient before $\mathrm{x}(0.201805,0.168749$ and 0.149001$)$ in the equation of approximating curve represents the rate of particle deposition, $\mathrm{cm} /$ day.

To measure the rate of formation of the supramolecular structure, the deposition of particles was carried out in glass tubes with a narrowing in the lower part, which reduced the area (concentration of suspension) by about 80 times. This mode of formation of the supramolecular structure is characterized by a constant increased content of the dispersed phase, unlike deposition in tubes with a constant cross section, where the formation of region with a high content of particles is limited by their deposition rate. The obtained data on the rate of formation of a supramolecularly ordered structure are presented in Fig. 2. At such rates one monolayer of silica particles with radius $158 \mathrm{~nm}$ is formed approximately within $90 \mathrm{~s}$. 


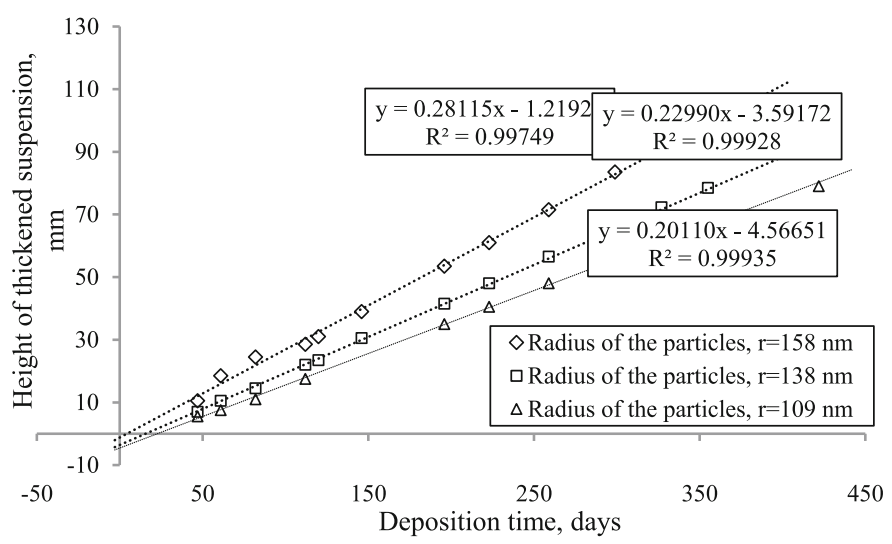

Fig. 2. Dependence of height of a supramolecular structure formed from silica particles of different size over time. The coefficient before " $x$ " $(0.28115,0.22990$ and 0.20110$)$ in the equation of approximating curve is a growth rate of the supramolecular structure in conditions of high concentration (excess) of the dispersed phase, mm/day.

\section{Results and Discussion}

Our data testify to that the particle deposition rate (Fig. 1) is linear and starts at the origin and strictly obeys the Stokes equation throughout the time. In turn, the analysis of data on the formation rate of the supramolecular structure (Fig. 2) shows that the formation rate of the ordered structure is also linear, however, the process of supramolecular crystallization does not begin immediately, but after some time, due to creation of "supersaturation" or some increased concentration of particles of dispersed phase in the bottom region. This time depends on size of particles and increases with their decrease.

As a result of deposition of monodisperse silica particles and formation of a supramolecular structure we noted that initially a zone with an increased concentration of dispersed phase was formed in the bottom region. The boundaries of this zone were clearly expressed, it possessed a constant height, both in the process of the beginning of deposition of particles, and after its completion. The height of the condensed

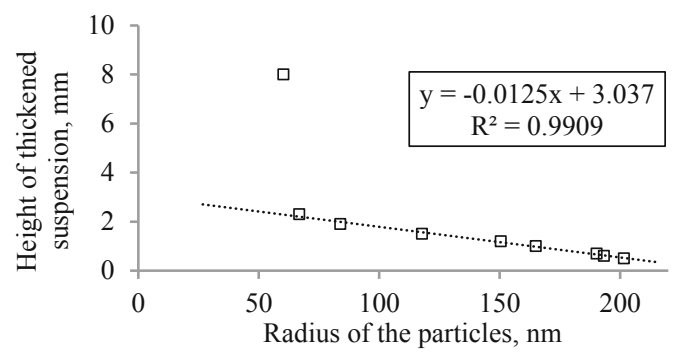

Fig. 3. Dependence of thickened suspension height on the particle size of the disperse phase after completion of deposition. 
suspension zone after deposition of particles was constant and depended only on size of dispersed particles (Fig. 3) with the exception of particles of radius less than $60 \mathrm{~nm}$. In this case a similar zone with clearly defined boundaries was not formed, and the supramolecular structure was not formed.

\section{Conclusions}

The obtained experimental data on deposition rate of monodisperse spherical silica particles with formation of a supramolecular structure showed that its formation was the result of a kind of second-order phase transition (self-organization of particles). This transition was associated with a threshold concentration of particles of dispersed phase in the area of deposition, below which the formation of the supramolecular structure did not occur. When this value was exceeded, the process of formation of a supramolecular structure started, and the rate of its formation was strictly linear and independent of the concentration of the dispersed phase. If for some reason the threshold concentration of particles was not reached (for example, very small sizes, low density of particles or high viscosity of the dispersion medium), then the formation of a supramolecular structure did not occur.

Acknowledgements. The work was accomplished with partial financial support by UB RAS program No. 15-18-5-44, and RFBR No. 19-05-00460a.

\section{References}

Deniskina ND, Kalinin DV, Kazantseva LK (1987) Noble opals. Novosibirsk, Nauka, Sib. otd. (Trudy institute geologii I geofiziki) 693, p 184

Kamashev DV (2012) Synthesis, features and model of formation of supramolecular silica structures. Phys Chem Glass 38(3):69-80

Stober W, Fink A, Bohn E (1968) Controlled growth of monodisperse silica spheres in the micron size range. J Colloid Interface Sci 26:62-69

Open Access This chapter is licensed under the terms of the Creative Commons Attribution 4.0 International License (http://creativecommons.org/licenses/by/4.0/), which permits use, sharing, adaptation, distribution and reproduction in any medium or format, as long as you give appropriate credit to the original author(s) and the source, provide a link to the Creative Commons license and indicate if changes were made.

The images or other third party material in this chapter are included in the chapter's Creative Commons license, unless indicated otherwise in a credit line to the material. If material is not included in the chapter's Creative Commons license and your intended use is not permitted by statutory regulation or exceeds the permitted use, you will need to obtain permission directly from the copyright holder.

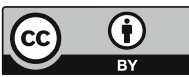

